\title{
De la liberté religieuse et du multiculturalisme aux États-Unis
}

L'exemple des Yoruba... Une lecture décaléeNathalie LucaÀ propos de : Stefania CAPONE, Les Yoruba du Nouveau Monde. Religion, ethnicité et nationalisme noir aux États-Unis, Paris, Karthala, 2005.

Nathalie Luca

\section{OpenEdition}

Journals

Édition électronique

URL : http://journals.openedition.org/assr/11323

DOI : $10.4000 /$ assr. 11323

ISSN : $1777-5825$

Éditeur

Éditions de l'EHESS

Édition imprimée

Date de publication : 1 décembre 2007

Pagination : 133-144

ISBN : 978-2-7132-2145-3

ISSN : 0335-5985

Référence électronique

Nathalie Luca, «De la liberté religieuse et du multiculturalisme aux États-Unis », Archives de sciences sociales des religions [En ligne], 140 | octobre - décembre 2007, mis en ligne le 02 juillet 2008, consulté le 19 avril 2019. URL : http://journals.openedition.org/assr/11323; DOI : 10.4000/assr.11323 


\title{
Nathalie Luca
}

\section{De la liberté religieuse et du multiculturalisme aux États-Unis}

\section{L'exemple des Yoruba... Une lecture décalée}

\author{
À propos de : \\ Stefania CaPONe, Les Yoruba du Nouveau Monde. Religion, ethnicité \\ et nationalisme noir aux États-Unis, Paris, Karthala, 2005.
}

\section{Liberté de religion et religion civile aux États-Unis}

Les États-Unis sont considérés comme un pays pluriculturel, très respectueux de la liberté religieuse. Cette image est en partie vraie aujourd'hui. Pour se construire, la nation américaine a néanmoins eu recours à l'élaboration d'une religion civile qui a limité l'application de cette liberté et retardé la réalisation d'un vrai pluriculturalisme. Jusque dans les années 1960 en effet, la « diversité religieuse américaine " se comptait essentiellement en nombre de "dénominations protestantes ». Aujourd'hui encore, les protestants représentent « $60 \%$ de la population américaine totale » (Fath, 2004 : 37). Cette forte prégnance du protestantisme aux États-Unis s'explique en partie par le mythe fondateur américain qui a lié, dès l'origine, la communauté citoyenne des États-Unis autour de l'image biblique du désert, comme lieu d'instauration du paradis terrestre : la communauté américaine s'est retrouvée unie par une mission honorifique : bâtir, sur ce sol conquis vers lequel Dieu l'avait guidée, la nouvelle Jérusalem (Bellah, 1975). C'était son devoir : le devoir d'un peuple élu. La « religion civile » américaine reposa avant tout sur une culture religieuse WASP - White, Anglo-Saxon Protestant. Elle fut consolidée par la fête de Thanksgiving (commémoration du grand repas de réjouissance partagé en 1621, après les récoltes, par les pères pèlerins puritains venus d'Angleterre et les Indiens Wampanoag), par le "serment au drapeau " (fait par chaque écolier qui jure allégeance à " une nation devant Dieu »), par l'optimisme ( « la mission que Dieu confère à l'Amérique constitu[ant] la garantie même du succès »), par la valorisation de la réussite dans le monde du travail (la puissance économique des États-Unis démontrant leur élection) ${ }^{1}$. Enfin, la

1. Sur la religion civile des États-Unis, voir, Fath, 2004, en particulier les pp. 54-60. 
religion civile américaine a fait du socialisme son ennemi. Bellah remarque ainsi que les États-Unis sont le seul pays industrialisé qui n'ait pas de mouvement socialiste significatif, bien que son organisation ait été légale et non censurée. La remise en cause de Dieu et de la religion, le ton athée et matérialiste du marxisme, le constat que les intellectuels européens, tout autant que la masse ouvrière se détournaient des églises et de la religion, ne pouvaient que déplaire aux Américains, les effrayer, compte tenu de l'inclusion de la croyance en Dieu dans leur pacte social. Pour finir, la guerre froide a fait de l'URSS un adversaire satanique auquel s'attaquait, selon les propos du président Reagan en personne, la «vertueuse » Amérique.

Les communautés culturelles ou religieuses qui remirent en cause ces éléments fondamentaux furent considérées par le peuple lui-même comme inadmissibles et condamnées au moindre écart par la justice : elles étaient une entrave à la réalisation du projet divin. Elles posaient à ce titre les limites implicites de la liberté religieuse pourtant proclamée de façon non restrictive par l'Article Premier de la Constitution américaine. Cela fut valable également pour quelques mouvements protestants contestataires. Annoncer, dans une attente toute apocalyptique, malheur à ce pays élu, comme ont pu le faire les Témoins de Jéhovah ou les Adventistes du Septième Jour, dénoncer la corruption des institutions séculières, censées être guidées par la main divine, comme se le permirent les Mormons, mettre en cause l'idéologie capitaliste, à la manière du Temple du Peuple, ont suscité des réactions sociales souvent violentes et conduit à la mise à l'écart des associations coupables, considérées comme des sectes. Leur intégration nécessita qu'elles mettent de côté ce qui apparaissait comme insupportable au peuple américain. Leur entêtement eut au contraire des conséquences tragiques, dont l'affaire du Temple du Peuple ou, plus récemment, celle de la communauté du Mont Carmel, sont de tristes exemples. Peu de mouvements chrétiens cependant mettaient en cause la religion civile américaine. Celle-ci par contre, ne pouvait être assimilée par les indigènes ou les Noirs Africains.

Lorsque, dans la Constitution américaine, les pères fondateurs ont inscrit la liberté religieuse et l'interdiction pour l'État d'intervenir en la matière, la compréhension de cette liberté était d'ores et déjà biaisée par la certitude que la barbarie devait être détruite et, par conséquent, par l'incapacité des autorités à imaginer que puisse être justifiée l'appellation de culture ou de religion pour des pratiques situées hors du champ de leur appréhension européenne de ces notions. Bellah rappelle que le " contrat " sous-tendant la religion civile américaine, conclu entre Dieu et les États-Unis, et entre les citoyens, impliquait la séparation des bons et des pécheurs. Elle avait débuté par le départ d'Europe des élus vers le nouveau continent. Elle devait se poursuivre par le repêchage des "fauteurs » vivant sur le sol divin. Ces «fauteurs » furent très vite identifiés par le prisme de leur appartenance ethnique. Les Noirs et les Indiens en furent les premiers représentants. Il apparut légitime de s'attaquer à eux violemment : il fallait empêcher les desseins 
sataniques de se réaliser. En les éliminant ou en les dominant, les « élus » protégeaient les fondations du « royaume divin » de leur anéantissement par l'intrusion de «puissances diaboliques ». Même les plus convaincus de la valeur fondatrice de la liberté religieuse dans la construction des États-Unis ne parvenaient pas à voir dans les croyances et pratiques noires ou indiennes un quelconque rapport avec une culture ou une religion. Chacune était plutôt le signe de leurs âmes perdues. Stefania Capone montre combien les tentatives des Afro-américains de résister à la religion civile ont été fermement surveillées et réprimées. Elles ont finalement abouti à l'émergence d'un communautarisme qui peut être lu, en soi, comme un exemple de dérive ou de limite du multiculturalisme. Précisons d'emblée que la lecture que je propose ici des Yoruba du Nouveau Monde est tout à fait décalée par rapport à la problématique de l'auteur. L'anthropologue met en scène, à travers l'exemple d'une communauté transnationale, la reconstruction, par les Afro-américains, d'une identité collective noire passant par la ré-affiliation avec des lignages africains imaginés. Pour ma part, j'ai cherché à détecter, à travers les remarquables descriptions et analyses de l'auteur, des éléments me permettant d'appréhender la régulation de la communauté afro-américaine par les autorités américaines ainsi que les limites de son intégration dans le système pluriculturel américain. Il ne s'agit donc pas, à proprement parler de proposer une note critique de l'ouvrage référencé ${ }^{2}$ mais d'utiliser ce travail pour rebondir sur un autre sujet de recherche : les limites de la liberté religieuse et les défis du multiculturalisme aux États-Unis.

\section{L'appropriation du récit biblique par les Afro-américains}

Les Afro-américains surent s'approprier le récit biblique pour donner sens à leur maltraitance. Ils découvrirent le christianisme, grâce au Grand Réveil protestant qui se développa durant la seconde moitié du XVIII siècle. Des pasteurs méthodistes, des quakers, des baptistes développèrent une conviction anti-esclavagiste et se firent un devoir de convertir les Noirs. Ceux-ci fondèrent bientôt leurs propres Églises grâce auxquelles les spirituals allaient créer une culture afro-américaine de résistance. Ils virent dans les États-Unis la nouvelle Égypte et comparèrent les hommes d'État américains aux pharaons égyptiens. Le peuple noir, réduit en esclavage, s'élevait ainsi au-dessus de l'oppresseur pour s'ériger en nouveau peuple élu. L'esclavage se para d'une vocation divine. Les Noirs ont ainsi cherché, tout au long du XIX ${ }^{\mathrm{e}}$ siècle et au-delà, le moyen de mettre à distance l'image négative que les Blancs avaient propagée. Cela passait par une recherche d'identité et une revendication culturelle qui les séparaient de la culture dominante chrétienne blanche et anglo-saxonne, allant parfois jusqu'à créer un dieu noir bien distinct.

2. Voir dans le Bulletin bibliographique (140-14) la recension par Terry Rey de l'ouvrage de S. Capone. 
Les leaders noirs, identifiés au Moïse des temps nouveaux, se multiplièrent lors du premier exode rural de 1879. Dans les années 1960, ils cherchèrent finalement à créer des villes noires, voire même un territoire indépendant : la République de Nouvelle Afrique (Capone : 57). Ces mouvements nationalistes furent réprimés par un programme du FBI : le COINTEL (Counterintelligence Program Black Nationalist-Hate Groups) et leurs protagonistes lourdement condamnés.

Dans sa forme la plus extrême, la résistance face à la culture dominante s'est traduite par le rejet du christianisme, considéré "comme une fausse religion » (Capone: 64). La naissance d'un islam noir, telle qu'on le retrouve dans la Nation of Islam, créée par Wallace Fard Muhammad et rendue célèbre par Elijah Poole, dit Muhammad, est à situer dans ce contexte. Il se donnait pour but d' " incorporer une doctrine raciale capable d'aider les Noirs, la race "originale", à dépasser leur position subordonnée à l'oppresseur blanc "satanique" » (Moore, 1986 : 191). Nation of Islam devint l'un des plus importants nouveaux mouvements religieux qui se soient développés au $\mathrm{XX}^{\mathrm{e}}$ siècle. Les adeptes croyaient qu'une bataille finale, " la bataille d'Armaguédon » porterait le coup fatal à la race WASP, présumée satanique. Auparavant, il fallait obtenir à tout prix un État indépendant et économiquement autosuffisant grâce auquel pourrait s'accomplir «le destin supérieur du peuple noir »(Capone : 77). Les adeptes évoluaient sur un tracé opposé à celui de la religion civile nationale. Ils la retournaient contre elle-même. « Les leaders musulmans furent dénoncés en tant qu'imposteurs religieux qui réclamaient la gloire pour eux-mêmes et détruisaient le christianisme. Ils furent accusés de sponsoriser un isolationnisme économique qui provoquait d'inutiles animosités avec leurs voisins. [...]. Il fut dit qu'ils étaient déloyaux, qu'ils complotaient contre le gouvernement des États-Unis et qu'ils contrôlaient chaque conduite de leurs membres » (Moore, 1986 : 192). Ce type de mouvement, mis sous haute surveillance, entrait pleinement dans la définition dialectique wébéro-troeltschienne de la secte, bien qu'employée sur un autre terrain que celui auquel elle s'applique généralement : il s'agissait en effet d'un groupe se positionnant en rupture avec une société dont il renie toute légitimité, précisément parce qu'elle s'est détournée de la volonté de Dieu, pour accomplir l'œuvre du diable. Cette posture fut difficilement tenable sur le long terme. Après la mort d'Elijah Muhammad, en 1972, «le mouvement prit le nom de Muslim American Society et rejeta la philosophie nationaliste qui avait caractérisé la Nation of Islam»(Capone : 80). Moins suivie, celle-ci demeure néanmoins active, tout comme la nouvelle Nation of Islam, refondée en 1978 après une scission et dirigée depuis par Louis Farrakhan. La volonté noire de revendiquer une communauté d'adhésion entièrement séparée de la culture WASP allait également se manifester sous l'influence de la culture yoruba. 
De la liberté Religieuse et du multiculturalisme aux États-Unis - I 37

\section{Le communautarisme yoruba}

Les années 1960 s'orientèrent vers la reconnaissance d'une ethnicité ouverte. Les États-Unis choisissent d'assumer, en l'intégrant, leur diversité culturelle. En 1965 en effet, la loi de 1924, interdisant "l'immigration d'étrangers reconnus inaptes à la citoyenneté »(Lacorne : 1997: 149), fut amendée en des termes reconnaissant l'égalité d'origine des immigrants. En cela, estime Alain Dieckhoff, ils affirment leur " destinée spécifique comme "laboratoire d'une nouvelle humanité" " (2000 : 94), une humanité liée, bien que plurielle ; une humanité fondée sur un "multiculturalisme civique " (Lacorne : $1997: 250$ sq.), c'est-à-dire, sur l'apprentissage de la diversité et des valeurs partagées. Plus tard, la mise en place de cette nouvelle logique citoyenne allait prendre la forme d'une politique publique de traitement préférentiel (affirmative action) favorable à l'intégration des Noirs et qui devait permettre aux universités de former " des élites à l'image des "nombreux peuples américains" ». Depuis, la nation américaine tente de devenir une vraie "fédération de peuples" (idem: 313-314). Cette nouvelle définition de la communauté américaine imaginée, pour reprendre l'expression d'Anderson, s'est concrétisée par des rituels fédérateurs. Denis Lacorne voit ainsi dans « les nombreuses célébrations de la diversité ethnique organisées chaque année par les universités américaines [autre chose qu'une] mode passagère ». Pour lui, ce sont «bien plutôt des célébrations civiques, le moyen rituel de rappeler et de glorifier la légitimité constitutionnelle de l'affirmative action, décrétée par les juges de la Cour suprême»(p. 314).

Cette "fédération de peuples » demeure, bien sûr, un idéal difficilement accessible. L'émergence de la communauté yoruba en montre les limites. Pour le comprendre, il faut bien préciser que la loi de 1965 n'a pas mis fin à la politique de ségrégation raciale. Les Noirs, exclus, ne pouvaient se construire que dans l'altérité. La création de la communauté yoruba aux États-Unis résulte de la volonté des Afro-américains de redécouvrir une culture originelle dont ils avaient tout oublié. Ils réinventèrent l'âme de l'Afrique telle qu'ils l'imaginaient avant qu'elle ne fût polluée par la colonisation. Héritage culturel, identification à un pays natal, apprentissage d'une langue maternelle, découverte des traditions et de la religion originelles leur permirent de se recréer un passé d'immigrés que l'histoire de l'esclavage leur avait volé.

La découverte de cette âme africaine passa par les voyages que les intellectuels et artistes noirs firent en Caraïbe et en Amérique latine : en Haïti, au Brésil ou à Cuba, partout où les anciens esclaves avaient su sauvegarder leur culture. Elle se manifestait dans le vodou haïtien, la santería cubaine ou le candomblé brésilien. Le nationalisme noir s'enveloppa alors d'une dimension religieuse non monothéiste qui devint primordiale. Les vagues d'immigrations cubaines permirent par la suite l'importation de cette dimension religieuse. Les Africains d'Amérique du Nord commencèrent à se faire initier. Mais la quête de la terre originelle n'en fut pas pour autant satisfaite. La santería, tout comme le candomblé et le vodou, 
avait subi l'influence religieuse des Blancs. Les Cubains n'y voyaient pas une religion africaine. C'était leur religion : une religion qui les avait accompagnés dans leur évolution, dans leur transformation, et qui n'avait rien du produit précolonial fossilisé que recherchaient les Noirs étasuniens. À ce malentendu, s'ajoutaient des tensions dues aux divergences d'intérêts des deux populations. Les Cubains étaient des réfugiés, accueillis par la société américaine parce qu'ils fuyaient le régime de Castro contre lequel luttaient les États-Unis. Pour cette raison, ils apparaissaient comme une minorité "symbole de la supériorité du capitalisme sur le communisme » (p. 118), dont il fallait faciliter l'intégration. Ils reçurent donc d'importantes aides gouvernementales et bénéficièrent, de surcroît, d'un matraquage médiatique très favorable à la reconnaissance de leur culture. Contrairement aux Afro-américains, ils n'étaient donc pas à la recherche de leurs origines, ni ne souffraient d'un rejet de la société d'accueil. Ils ne partageaient aucune des préoccupations des nationalistes noirs. Ces derniers poursuivirent leur quête en Afrique. Ils en rapportèrent un héritage yoruba qui leur donna une terre de naissance à laquelle se raccrocher : le Nigeria.

Le peuple yoruba regroupe à l'origine différentes ethnies présentes dans le sud-ouest nigérian. Son histoire commence dans les années 1820, au moment du déclin du royaume d'Oyo, "l'authentique Yoruba ", qui avait pendant longtemps dominé une bonne moitié de la région. Le pays yoruba s'étendait de la Volta, à l'Est, à la vallée du Niger, à l'Ouest. Les populations qui y vivaient étaient en bien des points distinctes et ne se concevaient pas comme un peuple uni. Elles se reconnaissaient davantage par leur appartenance locale, villageoise ou citadine. Trois facteurs ont joué dans leur unification. Tout d'abord, les missionnaires présents sur le territoire. Leurs sociétés, socialement très impliquées, ont su toucher, par leurs messages d'émancipation, la population la moins intégrée au système socio-politique de cette région. Par la suite, la colonisation, deuxième élément, permet la récupération massive du message chrétien, y compris par les élites autochtones écartées du pouvoir. Ce message contribue enfin, troisième élément, au développement endogène des sociétés africaines, dont la volonté nationaliste exige d'elles un effort de réappropriation des traditions leur permettant de s'inscrire en continuité avec l'époque précoloniale. Le terme de Yoruba, utilisé à la base par les missionnaires pour regrouper des ethnies pouvant communiquer entre elles par le biais d'un langage similaire, est alors récupéré par le peuple lui-même ${ }^{3}$.

Le fait que le christianisme ait joué un rôle primordial dans la formation du peuple yoruba auquel la colonisation donna naissance ne pouvait que contrarier les Afro-américains dont la soif de pureté et de non contamination de la culture occidentale restait inapaisée. Aussi se donnèrent-ils pour mission divine de rétablir le royaume d'Oyo, tel qu'il était originellement. Ce rétablissement se fit dans un village de Caroline du Sud. En 1970, Walter Eugène King, dit Adelabu

3. À ce sujet, voir Peel, 2000. 
Efuntola Adefunmi I, co-ministre de la culture du gouvernement provisoire de la République de Nouvelle Afrique, créa le premier village africain consacré au culte des dieux africains. Le propriétaire des lieux obtint d'abord l'expulsion de la communauté sous prétexte qu'elle ne cultivait pas ses terres. Celle-ci acheta donc un autre terrain et y construisit le village d'Oyotunji - en Yoruba, restauration d'Oyo - dont Adefunmi fut couronné roi.

Même si les données présentées par Stefania Capone ne permettent pas de l'affirmer, il semblerait que ce village n'ait pas fait l'objet d'une surveillance trop étroite du FBI. Rappelons rapidement le contexte : des groupes paramilitaires antiaméricains, comme les Black Panthers, de plus en plus sensibles à la politique de ségrégation raciale, sévissaient en même temps que se multipliaient les manifestations contre la guerre du Vietnam et les différentes émeutes sociales dues au mauvais climat économique. Dans cette situation de crise, les Afro-américains qui recherchaient pacifiquement leurs racines africaines n'étaient pas la priorité de l'État. Certes, ce village suscita la vigilance des autorités. Son installation ne se fit pas sans problèmes. Son indépendance finit néanmoins par être en partie reconnue par l'État de Caroline du Sud. Ils reçurent quelques aides gouvernementales et purent ouvrir leur propre école, même s'ils durent, pour cela, l'adapter aux exigences des autorités locales qui menacèrent plusieurs fois de la fermer ${ }^{4}$. Le village avait son drapeau, le drapeau du nationalisme noir (rouge, jaune, vert) et les élèves n'avaient pas à saluer le drapeau américain, rituel important dans l'entretien du sentiment national. La communauté restaura la polygamie, si récriée chez les Mormons. Elle promulga l'Ujima, le travail collectif, une forme de "socialisme africain » décrit comme « un monde d'harmonie, d'égalité et de fraternité " (Capone : 149), non sans lien avec les valeurs prônées par le Temple du Peuple. Il n'est pas inintéressant de souligner à cet égard que le Parti communiste américain des années 1930 avait soutenu le nationalisme noir, allant jusqu'à défendre " la naissance d'un $49^{\mathrm{e}}$ État américain qui devait rassembler les Noirs du Sud dans une république indépendante»(Capone: 95). De nombreuses études ont montré que, dès la fin $\mathrm{du} \mathrm{XIX}^{\mathrm{e}}$ siècle, les immigrés étaient critiqués dès lors qu'ils conservaient leur langue, leurs coutumes et leur religion d'origine. Pour s'intégrer, ils étaient invités à rompre le cordon ombilical avec leur terre de naissance, ce qui fut valable également pour les catholiques européens non anglophones. Ici, c'est tout ce monde linguistique, culturel et religieux qui est mis en place afin de créer un lien ombilical imaginaire avec la terre d'origine : la terre nigériane. Vêtements africains, coiffures afro, étude des langues africaines sont au cœur de l'expérience villageoise : le village d'Oyotunji se conçoit comme une région du Nigeria. Les Yoruba américains s’inventent ainsi une double nationalité, d'autant plus fictive qu'elle n'est pas reconnue par le Nigeria. Elle se concrétise par des rituels qui permettent non seulement aux initiés d'acquérir un nom

4. Cette information vient d'une collègue de Stefania Capone, Kamari Maxine Clarke, qui a publié un ouvrage sur Oyotunji Village. 
yoruba mais aussi de connaître les ancêtres qui les lient, de par leur sang, au sol africain. L'arbre généalogique est ainsi rétabli et permet d'élaborer une véritable religion identitaire ethnique.

$\mathrm{Vu}$ le contexte social rapidement rappelé, l'État américain avait tout intérêt à laisser se construire, à reconnaître voire à favoriser le développement de ce village, certes ouvertement anti-américain, mais à la fois pacifique et aux frontières clairement délimitées. Son isolement aidait en définitive la protection du reste de la communauté nationale. Sa dimension fondamentalement ethnique, absente chez les groupes sectaires qui ont suscité la vigilance des autorités américaines et l'inquiétude de la population, empêchait efficacement que ses valeurs se propagent au sein de la société WASP. L'exemple de cette communauté yoruba permet, en deuxième lecture, d'appréhender concrètement comment le communautarisme - dérive possible du multiculturalisme - peut conduire à la circonscription d'un cercle difficilement dépassable mais relativement préservé de l'ingérence des autorités.

Pourtant, Stefania Capone note que les familles avaient très mal pris le départ des leurs pour ce village. "À l'époque de la fondation d'Oyotunji Village, la plupart de ses résidants appartenaient à la première génération de "Yoruba" et leurs familles n'étaient pas directement engagées dans ce processus de redécouverte culturelle. Cela semble être une constante si l'on considère que, en 1989, un grand nombre d'habitants du village avaient rejoint la communauté contre la volonté de leurs familles " (Capone : 165). Les conditions de vie étaient plus que rudimentaires, la volonté de faire revivre les traditions révolues des ancêtres ayant amené les adeptes à se passer de l'électricité, du chauffage et de l'eau courante. Les mineurs vivaient ainsi dans un microcosme duquel il leur serait sans doute difficile de s'extraire. Enfin, l'insertion dans le village passait par un serment de fidélité aux rois, aux chefs et à la tradition yoruba. Même si cela reste à vérifier, il est néanmoins peu probable que les associations de protection des victimes de sectes se soient associées aux inquiétudes de ces familles. D'abord parce que ces dernières n'auraient sans doute pas dénoncé le choix de leurs enfants d'expérimenter un rêve qu'elles partageaient. Ensuite, parce que les associations dites « antisectes » se soucient, presque par définition, des mouvements susceptibles de propagation, et non pas des problèmes d'adaptation de populations ethnoculturelles minoritaires. Ainsi, lorsque le multiculturalisme conduit à la séparation de communautés ethnoculturelles, le religieux contestataire apparaît moins prosélyte, et par conséquent moins menaçant pour le maintien de la religion civile.

Lorsque l'on voit les dérives auxquelles a conduit l'intervention des autorités auprès des "sectes ", que ce soit dans l'affaire du Temple du Peuple ou dans celle du Mont Carmel, il est de surcroît intéressant de constater que, sans élément de résistance, le village d'Oyotunji alla jusqu'au bout de sa logique puis se transforma tranquillement. Pour assumer pleinement son indépendance, il décida de refuser 
l'aide de l'État américain. La survie du groupe obligea donc les adeptes à partir travailler en ville, où ils s'installèrent finalement. Il ne resta bientôt, au village, que les grands chefs de culte et leur roi, en tout, une vingtaine de résidants. Le village acquit l'eau, l'électricité et la télévision, laissant la société imaginée être pénétrée par la vie. Il devint un lieu sacré, lieu de pèlerinage où des Noirs venaient d'un peu partout se faire initier, rencontrer leurs ancêtres, leurs origines, leurs traditions et leurs dieux : s'enraciner avant de repartir chez eux. Grâce à cette province autoproclamée nigériane en plein cœur de la Caroline du Sud, on peut croire que les Afro-américains ont pu donner sens à leur trait d'union, et qu'ils peuvent désormais devenir, comme les autres immigrés, des individus se réclamant d'une double appartenance, d'une double culture assumée et maîtrisée, même si pour cela, une réinvention fut nécessaire.

\section{Autres limites du multiculturalisme}

On peut également se demander si les conflits avec la communauté yoruba ne furent pas limités par la parfaite étrangeté des traditions et pratiques yoruba pour la société américaine majoritaire. On peut supposer qu'elle ne se sentait pas concernée par la vie dans ce village pacifique isolé : le degré d'incongruence était tel qu'il pouvait la laisser indifférente. Des pratiques religieuses tranchant à ce point avec la culture chrétienne de la majorité des Américains posaient davantage de problèmes dans les villes. C'est un des sujets abordés par Stefania Capone qui a retenu mon attention. La Church of the Lukumí Babalú Ayé (CLBA), créée par le Cubain Ernesto Pichardo à Miami en 1974, fut ainsi au cœur de tensions qui opposèrent chrétiens et santeros d'origine cubaine. Cette Église ne se donnait pas seulement pour but la pratique de la santería, mais aussi l'enseignement des religions afro-caribéennes en milieu universitaire, ainsi que dans différents milieux professionnels. Elle travaillait efficacement à faire tomber les idées préconçues qui circulaient sur ces religions. Elle fut d'ailleurs si efficace que « la renommée de la CLBA ne cessa de croître, au point qu'en 1987, lors de la visite du pape à Miami, la chaîne de télévision IBC présenta la CLBA comme étant le seul concurrent crédible de l'Église catholique chez les Hispaniques de la ville » (Capone : 307). Elle avait pourtant connu, dès son établissement «l'hostilité des voisins et des autorités municipales » (Capone : 308), qui réagirent, au début des années 1980, au danger de propagation de valeurs, de croyances et de pratiques religieuses supposées peu compatibles avec celles de la société dominante, et imaginées de surcroît comme étant partagées par l'ensemble de la population cubaine. Or les catholiques cubains n'entendaient pas être confondus avec ces «sauvages » d'un autre temps, indignes de la modernité occidentale au cœur de laquelle ils se trouvaient réunis.

Le soutien de la justice américaine alla d'abord aux chrétiens. Le sacrifice rituel d'animaux non destinés à la consommation, accompli en vue d'alimenter les 
divinités, servit la cause des catholiques cubains. Il fut considéré contraire à l'intérêt public, pouvant causer un " "dommage émotionnel” aux enfants qui assistaient à la cérémonie » (Capone : 311 ) et devait être interdit par les lois anti-cruauté. En mettant le sacrifice sur la sellette, la justice sensibilisa la population américaine sur « l'aspect des pratiques africaines qui [la] choquaient le plus », d'autant que " les restes des animaux sacrifiés [étaient] souvent abandonnés aux environs des grandes villes [...] et [étaient] à l'origine des plaintes déposées par les membres de la ASPCA, la Société américaine pour la prévention de la cruauté envers les animaux" (Capone : 310). Notons que cette méthode de sensibilisation de la population américaine avait déjà fait ses preuves dans un tout autre contexte. Un siècle plus tôt, la Cour suprême avait en effet utilisé avec efficacité la polygamie des Mormons (nullement généralisée) pour rendre impopulaire ce groupe qui dénonçait avec un certain succès la corruption des institutions séculières. Le durcissement des lois anti-polygamie menaça jusqu'à sa survie (Moore, 1986 : 42). Les autorités remportèrent ainsi ce bras de fer. L'installation et le développement des Mormons à Salt Lake City, dans l'Utah, se firent au prix de leur renoncement à leurs particularismes et à leurs critiques des institutions. Ils sont considérés au $\mathrm{XX}^{\mathrm{e}}$ siècle comme des citoyens modèles, désormais respectueux des autorités.

Après plusieurs attaques en justice, et parce qu'elle avait perdu l'ensemble des procès, la CLBA dut fermer. Mais l'affaire ne s'arrêta pas là et rebondit finalement dans le sens inverse. Au début des années 1990, alors que s'élaborait en Europe, et plus spécifiquement en France, la politique de lutte contre les sectes contre laquelle les États-Unis allaient s'élever, la CLBA devint finalement «le symbole de la lutte pour la liberté religieuse en Amérique " (Capone : 312); en 1993, « les neuf juges de la Cour suprême décidèrent, à l'unanimité, que le gouvernement ne pouvait pas promulguer de lois qui empêchaient des pratiques religieuses et qu'il devait respecter le premier Amendement de la Constitution » (Capone : 313). Par ce jugement, les États-Unis retrouvaient leur regard d'antan sur le reste du monde. Les rédacteurs de la Constitution américaine, sensibles à la répression en Europe, avaient institué la liberté religieuse et la séparation des Églises et de l'État en pensant essentiellement aux protestantismes, auxquels ils voulaient assurer le développement sans contrainte dont le vieux continent les privait. En cette fin du second millénaire, ils sembleraient qu'ils aient une conscience renouvelée de la diversité qu'implique cette liberté dont ils veulent à nouveau se faire les défenseurs. Face à la montée de l'intolérance qu'ils dénoncent en ce domaine, les États-Unis ont élargi leur capacité d'acceptation de la pluralité religieuse et ethnique et ont refait de sa promotion un axe de leur rôle dans le monde. C'est ainsi qu'il faut interpréter la création d'un rapport annuel sur la liberté religieuse fait au nom de l'International Religious Freedom Act, signé en 1998 par le Président américain et prenant le contre-pied des rapports de la Mission Interministérielle de Lutte contre les Sectes (la MILS), renommée depuis Mission Interministérielle de Vigilance et de Lutte contre les Dérives 
Sectaires (MIVILUDES), instituée en France à la même époque. Les religions afro-caribéennes, peu présentes sur le sol français, deviennent ainsi un des emblèmes possibles, mais inattendus, d'un vieux conflit franco-américain, réanimé depuis une quinzaine d'années par l'inquiétude que suscite un marché religieux dérégulé.

\section{Un maillage de défense international}

Les religions d'origine afro-américaines apprennent cependant à se défendre seules et se regroupent pour cela dans des organisations internationales. Entre le vodou, le candomblé, la santería et la religion yoruba, les cultes afro-caribéens et américains se sont développés en formant des maillages transnationaux éventuellement concurrents. Le mouvement yoruba est le premier à s'être organisé au niveau international. Il le fait déjà par l'intermédiaire d'une société civile internationale qui organise des conférences mondiales sur la tradition et la culture des Orisha (COMTOC). La première a eu lieu en 1981 dans la ville d'IléIfé au Nigeria. Elle aboutit, en 1997, à la création d'un Ethic Committee. Pour éviter que les communautés dont il se fait l'avocat se retrouvent impliquées dans des dérives qui porteraient atteintes à l'ensemble de ces traditions, il tente d'atteindre une forme d'orthodoxisation et d'institutionnalisation de pratiques à visées universelles, aptes à " "corriger" les prêtres et les prêtresses dont le comportement "incorrect", la soif de richesse, l'exploitation des fidèles pos[ai]ent des graves problèmes à la communauté de croyants» (Capone : 282). Dans une perspective toute différente, naquit également, à la toute fin des années 1990, le National African Religion Congress (NARC) « avec pour objectifs de : représenter les religions africaines au niveau national et international ; affirmer la liberté religieuse ; garantir le droit de réaliser les cérémonies et les rituels, incluant le sacrifice d'animaux ; assurer le droit d'effectuer des cérémonies publiques, lutter contre toute discrimination venant d'autres religions » (Capone : p. 301). Il s'agit d'une instance supérieure ayant pour rôle d'assurer la qualité des services offerts par ses adhérents (inscrits dans un bottin international certifié) et de les défendre contre des attaques extérieures. Sa reconnaissance lui permettrait de profiter des mêmes droits que les autres religions. Mais le NARC espère plus encore: il veut voir reconnue l'efficacité des rituels thérapeutiques et le bienfait des plantes utilisées par les prêtres et prêtresses. Bien sûr, il est encore loin de ses fins, mais cette union de prêtres venus des États-Unis, du Canada, des Caraibes, d'Amérique latine, d'Afrique, et même d'Europe (du Royaume Uni et d'Espagne) donne un poids nouveau à la religion des Orisha. Ainsi, la résistance à l'hégémonie chrétienne s'organise et jouera peut-être dans la redistribution de l'identité culturelle et religieuse d'individus et de nations qui se cherchent encore. Déjà, malgré leur caractère profondément ethnique et sans qu'aucun prosélytisme ne soit nécessaire, les religions afro-américaines initient des Occidentaux blancs... 